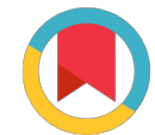

Check for updates

\title{
Drug respositioning by integrating known disease-gene and drug-target associations
}

\author{
Duc-Hau Le
}

Vinmec Research Institute of Stem Cell and Gene Technology

\begin{abstract}
Computational drug repositioning has been proven as a promising and efficient strategy for discovering new uses from existing drugs. To achieve this goal, a number of computational methods have been proposed, which are based on different data sources of drugs, diseases and different approaches. Depending on where the discovery of drug-disease relationships comes from, proposed computational methods can be categorized as either 'drug-based' or 'disease-based'. The proposed methods are usually based on an assumption that similar drugs can be used for similar diseases to identify new indications of drugs. Therefore, similarity between drugs and between diseases is usually used as inputs. In addition, known drug-disease associations are also needed for the methods. It should be noted that these associations are still not well established due to many of marketed drugs have been withdrawn and this could affect to outcome of the methods. In this study, instead of using the known drug-disease associations, we based on known disease-gene and drug-target associations. In addition, similarity between drugs measured by chemical structures of drug compounds and similarity between diseases sharing phenotypes are used. Then, a semi-supervised learning model, Regularized Least Square (RLS), which can exploit these information effectively, is used to find new uses of drugs. Experiment results demonstrate that our method, namely RLSDR, outperforms several state-of-the-art existing methods in terms of area under the ROC curve (AUC). Novel indications for a number of drugs are identified and validated by evidences from different resources.
\end{abstract}

\section{Keywords}

Drug repositioning, Regularized Least Square, RLSD

Copyright The Author(s) 2017. This article is published with open access by BioMedPress (BMP).

This article is distributed under the terms of the Creative Commons Attribution License (CC-BY 4.0) which permits any use, distribution, and reproduction in any medium, provided the original author(s) and the source are credited. 